\title{
Antenna beam forming and beam controlling for Improving the Wi-Fi signal
}

\author{
K. Raghavendra, A. Swetha, S. Naresh
}

\begin{abstract}
Antenna design plays a prominent role when we consider the far-field patterns when compared with near field patterns. By using a PIN diode (which has a wide intrinsic region when compared with normal diode) as a switch and a capacitor as a filter we are proposing an antenna design that able to radiate far field regions. The reason behind choosing capacitor as a filter means that we are designing antenna to transfer high frequency signals. For antenna deign simulations we have different software's (like FEKO, ZELAND IE 3D etc.,) but the preferred software for this antenna design is Computer Simulation Technology Microwave Studio (CST MWS) as we are operating in a frequency band of 2 to $2.54 \mathrm{GHz}$ and the diameter of the antenna $20 X 20 \mathrm{~cm}$. Based on the diameter chosen the antenna is able to switch direction of signalling by using a switch with four different angles. The material used for antenna design FR-4 which is a Composite material composed of fibre glass cloth with an epoxy resin blinder i.e. frame resistance and SMB connectors with $75 \Omega$ impedance.
\end{abstract}

Keywords ---- Antenna Directivity, Strip antenna array, Wi-Fi.

\section{INTRODUCTION}

If we want to increase the gain of the antenna as well as to reduce the unwanted source we must be able to control the beam of radiation pattern and this can be achieved by using the Beam Control antenna which is designed by using PIN diode as a switch and capacitor as filter. Beam antenna must work on the principle of directional antenna which has the ability to concentrate to a single direction with high gain and to reduce the energy that gets wasted in different areas (example Yagi antenna). The material used for antenna design FR-4 which is a Composite material composed of fibre glass cloth with an epoxy resin blinder i.e. frame resistance and SMB connectors with $75 \Omega$ impedance.

\section{ANTENNA DESIGN}

In this method we are using four PIN diodes as switches with four different angles to allow the specific radiation pattern. Each diode need two capacitors to act as filter in order to prevent the dc current not to enter the antenna[1] [3] If any one of the diode is supplied with energy of 5V DC then each angle is able to radiate 90 degrees. This antenna design is done by using FR-4 material whose dielectric substrate is assumed to be $0.17 \mathrm{~mm}$ and the thickness of copper wire is assumed to be $0.038 \mathrm{~mm}$. Here the pin diode which is acting

Revised Manuscript Received on 14 August, 2019.

K.Raghavendra, Asst. Professor, Dept. of ECE, Malla Reddy Engineering College for Women(Autonomous), Secunderabad-TS Email: raghu.siiet@gmail.com ${ }^{1}$,

Swetha, Asst. Professor, Sri Indu Institute of Engineering \& Technology, Ranga Reddy-TS swetha.viit@gmail.com

S. Naresh, Asst. Professor, Sri Indu Institute of Engineering \& Technology, Ranga Reddy-TS, surabunaresh2012@gmai.com as a switch restricts the DC current and allows the AC current to pass through the antenna. The Capacitor filter acts as a high pass circuit which allows only high frequency signals and avoids the low frequency signals [2].

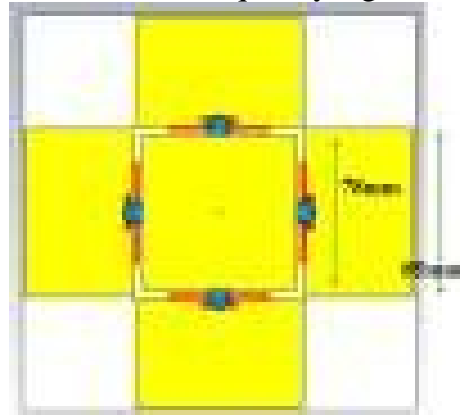

Fig 1: Antenna Front View

The front view of the antenna is represented in fig land the middle patch is simulate as $2.54 \mathrm{GHz}$ frequency with the diameter $76 \mathrm{~mm} \times 76 \mathrm{~mm}$ while the outside four patches is to generate the far-field and diode switching and filtering is used for antenna directivity.

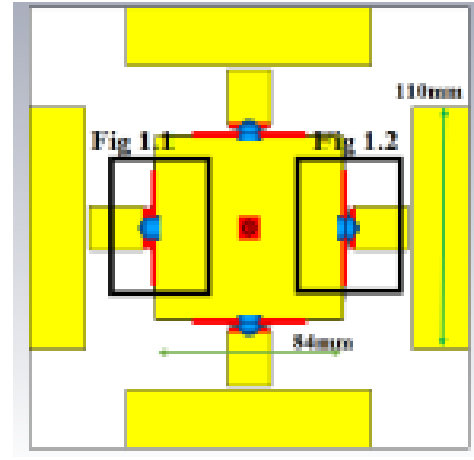

Fig: 2 Antenna Back view

The red square at the middle of the part in fig 1 indicates SMB connection with $75 \Omega$ impedance. This region shows the antenna connection from main section to ground. The mid region of the antenna is of diameter $86 \mathrm{~mm}$ X $86 \mathrm{~mm}$. In the design point of view the PIN diode is connected between the ground and the small patch of the antenna as shown below

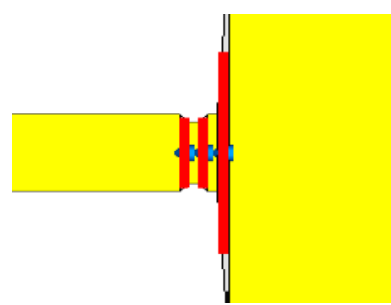

Fig: 3 Antenna Design with Switch as Short Circuit

Published By:

Blue Eyes Intelligence Engineering \& Sciences Publication 


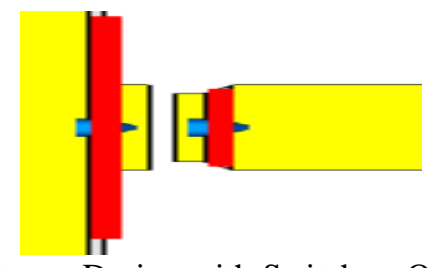

Fig: 4 Antenna Design with Switch as Open circuit

In fig 3 we can observe that the connection between the ground of the antenna and the small patch did not connect which shows that PIN diode is not connected i.e. switch is working as open circuit logic. Fig 2 and Fig 3 are the zoom versions of Fig 1.

In fig 1 we observe three capacitors are represented as three triangles with blue colour dots and the middle one is the PIN diode acts as switch whereas remaining three as filters. When we apply the DC current of 5V then PIN diode gets activated and it will drain the AC signal from antenna to other site and both the filters restrict the dc current.

From Fig 3 we can observe that if PIN diode is not connected then the signal from antenna unable to pass through the other side of antenna then it cannot generate the far field on the other site.

\section{RESULT ANALYSIS:}

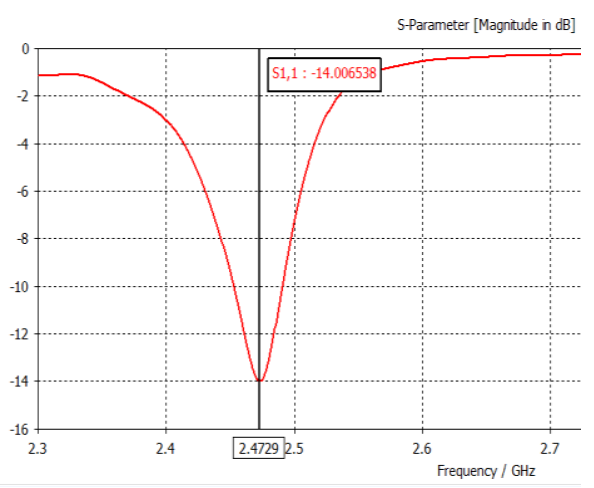

Fig: 4 S11 Parameter

From fig 4 we observe a frequency drop at $2.47 \mathrm{GHz}$ and $\mathrm{S} 11$ magnitude at -14.00 and after simulation with and without diode the frequency and S11 magnitude remained same. The desire frequency range is dropped to below -10 while the frequency $2.47 \mathrm{GHz}$ is under ISM band and in the range of WI-FI band range i.e. $2.4 \mathrm{GHz}$ to $2.5 \mathrm{GHz}$ [4].

\section{CONCLUSION}

The antenna is able to radiate far field signal by using diode and capacitor and by using CST MWS software we are able to simulate the antenna to get desired frequency, far-field and gain. As diode rotates it represents far-field with different angles.

\section{REFERENCES}

1. J.L.Lawson, Yagi-antenna Design, American Radio Relay League, 1886.

2. W. K. Chen, The Electrical Engineering Handbook, vol. 16. Academic Press, 2004

3. David M. Pozar, D. Schaubert, Microstrio Antenna,'IEEE Antenna and propogation Society, 1995

4. Stephen C. Thierauf, High Speed Circuit Board Signal Intergration, Artech House
5. N. Nithiyanandam, K. Venkatesh, M. Rajesh, Transfer The Levels Of The Monitored Carbon, Nitrogen Gases From The Industries, International Journal of Recent Technology and Engineering, Volume-7 Issue-6S3 April, 2019.

6. Sivanesh Kumar, A., Brittoraj, S., Rajesh, M., Implementation of RFID with internet of things, Journal of Recent Technology and Engineering, Volume-7 Issue-6S3 April, 2019.

7. Rajesh, M., Sairam, R., Big data and health care system using mlearningJournal of Recent Technology and Engineering, Volume-7 Issue-6S3 April, 2019.

8. Rajesh, M., and J. M. Gnanasekar. "Path Observation Based Physical Routing Protocol for Wireless Ad Hoc Networks." Wireless Personal Communications 97.1 (2017): 1267-1289. 